\title{
Care of the Patient after Renal Allograft Failure: Managing the Present and Planning for the Future
}

\author{
Richard Fuquay Isaac Teitelbaum \\ Division of Kidney Diseases and Hypertension, University of Colorado School of Medicine, Aurora, Colo., USA
}

\section{Key Words}

Kidney transplantation - Dialysis mortality - Dialysis trends . Immunosuppression $\cdot$ Nephrectomy $\cdot$ Peritoneal dialysis

\begin{abstract}
The number of patients with end-stage renal disease undergoing kidney transplantation - both cadaveric and livingdonor - continues to rise. With long-term graft survival relatively fixed, this trend means that increasing numbers of patients are returning to dialysis after graft loss. Most will never be retransplanted, which introduces a host of clinical questions regarding optimal management of this unique patient population. In this paper, we explore data that informs astute care of the patient requiring dialysis after graft loss. We address new data about the increased clinical risk and the optimal dialysis modality in renal allograft loss, explore new approaches to immunosuppression and transfusion management, and examine the risks and benefits of allograft nephrectomy and timing thereof. While there are no randomized clinical trials in this field, rapidly evolving data will aid the clinician whose practice includes patients who have been transplanted and are returning to dialysis.
\end{abstract}

Copyright $\odot 2012$ S. Karger AG, Basel
(C) 2012 S. Karger AG, Basel

0250-8095/12/0364-0348\$38.00/0

Fax +4161306 1234

E-Mail karger@karger.ch

www.karger.com
Accessible online at:

www.karger.com/ajn

\section{Introduction}

End-stage renal disease (ESRD) is a complex illness with myriad stages and therapeutic options. In 2001, Davies et al. [1] introduced the term 'integrated care' which conceptualizes hemodialysis (HD), peritoneal dialysis (PD), and transplantation as three complementary treatment modalities, each with advantages and disadvantages for an individual patient. Rather than viewing paradigm modality transition as a negative event described as 'technique failure', changes should be anticipated as usual events for patients living with a chronic disease. This inevitability of change is particularly true for patients with a renal allograft. While short-term survival and freedom from rejection have improved remarkably with the advent of modern immunosuppression, long-term survival has not shown the same improvement. Current data shows that graft attrition in the first 12 months after transplantation surgery has dropped markedly in the last two decades, while graft survival in later time periods has improved only modestly [2]. Thus, the need for dialysis after graft loss (DAGL) is among the top five reasons for initiation of dialysis [3], making this an important topic in clinical nephrology. Before examining management of immunologic risks in DAGL patients and the risks and 
benefits of allograft nephrectomy, we will explore the increased mortality and clinical risks experienced by DAGL patients and address the optimal dialytic modality in these patients.

\section{Do Patients Experience Increased Mortality after Failure of a Renal Allograft?}

The 2007 USRDS report estimates that $4.1 \%$ of incident dialysis patients have a failed allograft. That number will continue to increase as the number of annual renal transplants rises. Estimates of the proportion of incident dialysis patients that have a failed allograft vary by study. The vast majority of patients with a failed transplant require long-term dialysis as only $15 \%$ of patients with a failed allograft will be retransplanted [4].

DAGL is a transition period during which patients experience higher mortality than they do with even a poorly functioning allograft $[5,6]$. These patients have a 1-year mortality rate of $16 \%$ and a 3 -year mortality rate of $33 \%$ [7]. Most of the mortality is due to cardiac causes. Loss of renal allograft function at 12 months after transplantation has been found to correlate with cardiovascular survival in both DAGL patients and patients with a functioning allograft [8].

Overall mortality in DAGL populations has been studied in various settings with differing results. In a small 2001 single-center study from Toronto [9], patients starting PD with a failed transplant were found to have higher rates of death, peritonitis, catheter complications, and modality transfer than controls starting PD de novo. Interestingly, a similarly-sized single-center study from the UK [10] found that having a failed transplant was associated with better patient survival, but that association disappeared when the investigators corrected for age and comorbidities. A 2003 publication based on Baxter database patients [11] found that survival was better among PD patients with a failed graft than de novo PD patients; they did not adjust for comorbidities.

The most definitive studies on this topic come from national transplant databases and have been published in the last decade. When mortality of DAGL patients is compared to that of never-transplanted dialysis patients, selection of the comparison group is important. Rao et al. [12] compared Canadian DAGL patients to all dialysis patients in a 2005 publication and found equal survival. In a follow-up study published in 2007 [13], the same author compared DAGL patients to dialysis patients actively listed for a first renal transplant, and found $78 \%$ increased

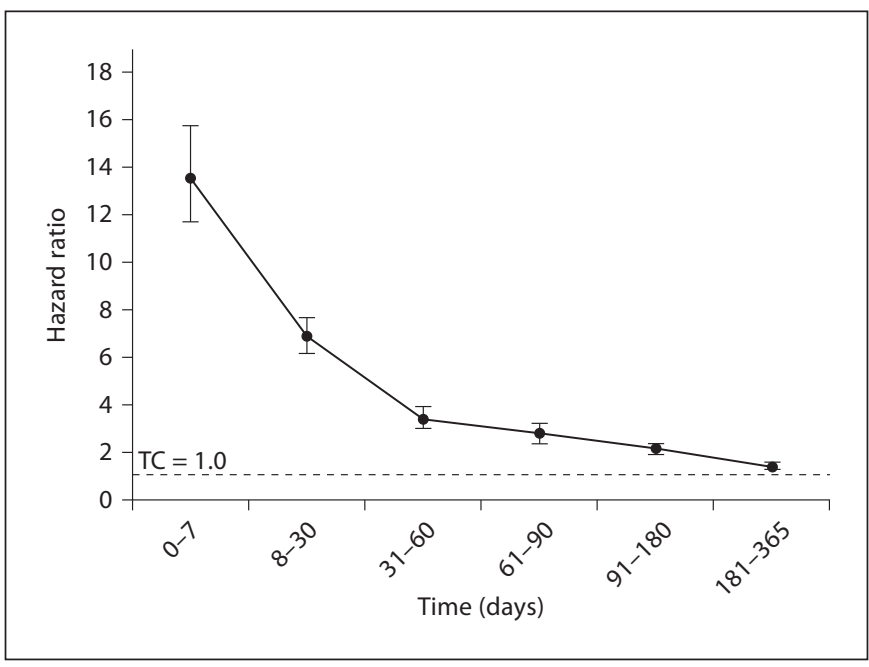

Fig. 1. Mortality hazard ratio for DAGL patients in the first year after graft failure, compared to transplant candidates receiving dialysis. The horizontal axis is time since graft failure, and the vertical axis is hazard ratio. In the first year after DAGL, patients experience higher mortality at all time points [from 13, with permission].

mortality in the DAGL patients. Most of the mortality burden was realized in the first 2 months after graft failure (fig. 1). This result is likely due to the fact that transplant candidates have fewer comorbidities than does a population of all dialysis patients. The latter of these two publications is more relevant when caring for an individual patient who has declining graft function, as that patient has a more favorable risk profile than does a member of the general dialysis population.

There are limited data regarding patient and treatment factors that may increase the risk of death after allograft failure. One publication [7] reported that white race, female gender, peripheral vascular disease, congestive heart failure (CHF), and failure of a first allograft are associated with particularly high mortality. In the same study, duration of graft function, depleting antibody induction, living versus cadaveric donor, and history of acute rejection were not associated with increased risk of death after graft failure. A recent study by Perl et al. [14] concluded that mortality in DAGL patients increases with the duration of allograft function, and is negatively associated with preemptive transplantation.

In summary, patients experience an increased risk of death in the days and weeks after returning to dialysis. There are a variety of contributing risk factors - which 
are primarily non-immunologic - and attention should be paid to risk factor reduction, which we will explore next.

\section{What Can Be Done to Reduce Mortality in DAGL Patients?}

There are a large number of dialysis patients, including DAGL patients, for whom a cause of death is not determined. In these patients, sudden cardiac death is a likely but not certain culprit. Of DAGL patients with a known cause of death, cardiovascular disease is the most common killer, followed by infections $[4,6,15]$. The prominence of cardiovascular disease is perhaps not surprising as the kidney transplant population is becoming older and more often diabetic. Mortality is particularly high among DAGL patients with diabetes [13]. In addition, there is evidence that DAGL patients do not receive CKD care that is considered standard among patients with failing native kidney function [16].

One such important area of patient care is optimization of cardiovascular risk factors, which are heightened in DAGL patients due to prolonged CKD vintage [17] and the adverse cardiovascular effects of immunosuppressant medications. DAGL patients often have compelling indications for specific medications. For example, patients with reduced left ventricular function or a history of stroke should receive an ACE inhibitor or ARB. Patients with a history of coronary artery disease should receive aspirin, a statin, and a $\beta$-blocker. All patients with a failing graft should be considered for statin therapy, particularly in light of the recent SHARP trial which has relevance not only for ESRD but all CKD patients [18].

While it is difficult to make a blanket recommendation regarding blood pressure targets in DAGL patients, attention should be paid to blood pressure and all modifiable cardiovascular risk factors. Registry data suggests that BP is not optimally managed in transplant patients, and that hypertension is associated with decreased graft survival [19]. Steroid and calcineurin inhibitors are known to raise blood pressure. Immunosuppressive drugs also raise the risk of post-transplant diabetes mellitus and should be minimized or discontinued in patients with no chance at repeat transplantation.

The second leading cause of death in DAGL patients, after cardiac causes, is infection [7]. Sepsis rates are nearly 3 -fold higher in failed transplant patients compared to incident $\mathrm{HD}$ patients on the transplant waiting list. HD was found to be a stronger risk factor for sepsis among
DAGL patients than was obesity, CHF, age, or even diabetes [20]. In a retrospective study of Medicare admission claims among DAGL patients, receiving HD as opposed to $\mathrm{PD}$ was associated with 1.7 -fold increased septicemia risk $(\mathrm{p}<0.001)$. Why might that be?

Central venous catheters in DAGL patients are associated with poor outcomes [21], and access planning is an opportunity for care improvement in this at-risk population. Furthermore, in arteriovenous grafts, immunosuppression confers a higher risk of access infection [22]. It appears from a Canadian study comparing transplant patients seen in a transplant clinic with non-transplant patients seen in a standard CKD clinic that patients with a failing allograft receive less attention to HD access creation, and when they do, it is at a lower eGFR [23].

This difference in care processes may be somewhat justifiable due, in part, to the slower rate of renal function loss in patients experiencing graft failure [17]. Furthermore, chronic inflammation likely decreases the primary patency rate of newly created HD accesses. However, an interesting single-center study from Poland has shown that about $50 \%$ of forearm arteriovenous fistulas thrombose in transplant patients with an average graft survival of 5 years. In these patients, reconstruction of a forearm fistula is possible, with primary and secondary patency rates that are only slightly worse than in ESRD patients without a failed graft [24].

In summary, cardiac risk factors should be aggressively treated in DAGL patients, as cardiovascular death is the most common cause of death in these patients. Infection is the second most common cause of death, and central venous catheters appear to be the greatest modifiable risk factor for infection in DAGL patients. Avoidance of central venous catheters now brings us to dialytic management of DAGL patients.

\section{Is There an Optimal Dialysis Modality for DAGL Patients?}

Data from both USRDS and the Canadian Organ Replacement Registry (CORR) indicate that PD use is falling in formerly transplanted patients. One possible reason is concern about the potential for a higher incidence of rapid transport kinetics in the peritoneal membranes of patients who have received an allograft, which may predispose to technique failure. The frequency of rapid transport kinetics was indeed increased in a small series of 19 PD patients with DAGL compared to de novo PD patients [25]. However, in a much larger Australian study 
of 5,746 total patients and 119 DAGL patients, the DAGL patients were not found to have different transport kinetics [26]. Furthermore, both a single-center English study [10] and a large American database study [11] found technique survival among DAGL and de novo PD patients to be equal. Furthermore, a study of 494 American DAGL patients treated with $\mathrm{PD}$ found equal technique survival when compared to both case-matched de novo PD patients and $\mathrm{PD}$ patients transferring from $\mathrm{HD}$ [27]. Though calcineurin inhibitors have a theoretical risk of altering transport states via profibrotic effects, this relationship has not been demonstrated [25]. Therefore it appears that the presence of a non-functioning renal graft does not increase the likelihood of rapid transport status.

Are there mortality data comparing PD- and HDtreated patients who have lost a renal allograft? Singlecenter studies demonstrate favorable [28] or equal [10] survival data in DAGL patients treated with PD compared to HD. Recent, more definitive data involving a larger number of patients from Perl et al. [14] shows that among Canadian DAGL patients there is no difference in survival between PD- and HD-treated patients. This study included 389 PD-treated and 1,721 HD-treated patients and found equal early and late survival. The authors hypothesize that the early survival advantage of de novo PD patients, which is often attributed to residual renal function (RRF), is attenuated by the more advanced degree of renal failure seen in DAGL patients [14, 23].

What is the optimal modality choice after failure of a renal allograft? Patient survival appears to be equal among PD- and HD-treated DAGL patients. The reported outcomes do not justify the observed decrease in the use of PD for patients with DAGL.

\section{What Is the Optimal Approach to Immunosuppression Management?}

Immunosuppression management after graft loss is an area of debate. There are data suggesting that immunosuppressed PD patients have a higher incidence of peritonitis and resulting need for catheter removal [9], which would support rapid tapering of immunosuppression. On the other hand, removal of immunosuppression with consequent loss of remaining allograft function may, in turn, drive more rapid loss of total RRF, adversely affecting patient outcomes. RRF is an important factor in decisions regarding immunosuppression management.

Indeed, a more rapid loss of RRF was reported in a small retrospective study [29] of 8 DAGL patients and 16

Care of the Patient after Renal Allograft

Failure de novo PD patients. In this study, RRF was similar at initiation of $\mathrm{PD}(8 \mathrm{ml} / \mathrm{min})$ but declined more rapidly in DAGL patients. Cyclosporine was tapered relatively rapidly - over a 3-month period - following initiation of PD, and the majority of patients in this series developed anuria. These data contrast with a larger Portuguese study of 148 patients showing that preservation of RRF is equivalent in DAGL patients and de novo PD patients [30]. In the Portuguese study, patients' antiproliferative drug was stopped abruptly, and the steroid and calcineurin inhibitors were tapered over 'several months'. RRF is likely to be equally well preserved in the DAGL population, provided careful and individualized attention is paid to the immunosuppressive regimen.

Jassal et al. [31] made use of decision analytic modeling (rather than empiric data) to address the question of whether continuation or cessation of immunosuppression is superior in DAGL patients on PD. The decision model was based on the assumptions that (1) residual GFR from a graft or from native kidneys confers equal mortality benefit and (2) cessation of immunosuppression would lead to risks of cancer and infection equal to that in the general population. Empiric risks of various infections and cancers, and average GFR decline, were imputed from observational studies (using modern immunosuppression) to inform the model. In their model, immunosuppression would be stopped when urine output fell below $100 \mathrm{ml} /$ day. The authors calculated a life expectancy of 5.3 years in non-immunosuppressed patients, and 5.8 years in immunosuppressed patients. This analysis, though intriguing in its methodology, did not account for post-transplant diabetes and all cardiovascular outcomes (the leading cause of death in this group). Nevertheless, the authors argue for routine continuation of immunosuppression in DAGL patients on PD, based on these data. To summarize, data is weak but generally supportive of the common clinical practice of continuing some amount of immunosuppression in DAGL patients.

\section{What Are Current Recommendations for Patients Needing Blood Products?}

About $85 \%$ of DAGL patients will not receive another kidney transplant. Minimizing sensitizing events to facilitate repeat transplantation should be a high clinical priority. A paradigm swing in transfusion practice occurred in the 1980s in response to HIV and viral hepatitis. Current practice is to transfuse only when medically necessary. Yet, one center reports that up to $45 \%$ of kid- 
ney transplant patients receive blood products in the month following transplantation [32]. Transfusion is likely to be associated with increased panel-reactive antibody (PRA), which is in turn associated with decreased graft survival [33]. Clinicians should be even more conservative with blood transfusions in patients with a failed kidney allograft, as they have a much heightened risk of sensitization compared to a patient wait-listed for his or her first kidney transplant [34].

Sensitization makes transplantation less likely and more burdensome. Scornik and Meier-Kriesche [34] convincingly argue that transfusions in patients with a previous organ transplant or pregnancy may be an avoidable source of sensitization that limits future transplants and complicates the care of these patients. They also describe how donor-specific transfusion and immunosuppression management could reduce the sensitization risk of transfusion. Thus, there is much room for improvement - and possibly new strategies altogether - to avoid the sensitization caused by blood transfusion and thereby to increase the likelihood of repeat transplantation. This issue requires careful clinical attention.

\section{In Summary - What Are the Risks and Benefits of Allograft Nephrectomy?}

Due to an absence of clear data on the subject, guidelines for which patients should undergo allograft nephrectomy, and at what time relative to resumption of dialysis, are lacking [35]. The potential benefits of allograft nephrectomy include removal of several harmful factors: a nidus of inflammation, the need for immunosuppression, and the presence of antigens that will raise the PRA over time and endanger future transplantation. Conversely, the risks of graft nephrectomy include the risks of an invasive surgical procedure, the loss of RRF, and the loss of potential immunosuppressive effects from the allograft itself.

Johnston et al. [36] report observational data from 19,107 USRDS patients with transplant failure showing that allograft nephrectomy was performed in $56 \%$ of patients experiencing graft failure within 12 months of transplantation, compared to only $27 \%$ of patients with at least 12 months of allograft function before graft failure $(\mathrm{p}<0.0001)$. Nephrectomy was associated with increased risk of death (HR 1.13, 95\% CI 1.01-1.26, p < 0.0001) in patients who experienced graft failure within 12 months of transplantation. In contrast, there was a negative association with death (HR 0.89, 95\% CI 0.83-0.95, p <
0.0001) in patients with later graft failure. An important clinical consideration is that nephrectomy is associated with adverse effects that are not benign. In the Johnston paper [36], the following 90-day complication rates were observed: myocardial infarction $1.1 \%$, CHR $7.5 \%$, sepsis $6.2 \%$, and death $4.7 \%$. Rates of sepsis and CHR (but not myocardial infarction or death) during the same hospitalization as the nephrectomy were significantly higher in patients with early graft loss compared to patients with late graft loss. All of these data are purely observational and one must remember that direct causality cannot be presumed in any of these relationships.

Additional retrospective USRDS data on allograft nephrectomy were published in 2010 [37]. The authors examined 10,951 patients in the USRDS who required DAGL in the decade commencing in 1994. In all, 3,451 (31.5\%) patients underwent allograft nephrectomy. Compared to patients who did not undergo nephrectomy, the nephrectomy-treated patients were slightly younger (by 4.6 years) with a lower likelihood of tobacco use, myocardial infarction, CHF, peripheral vascular disease, and other comorbidities. The nephrectomy-treated patients had reduced unadjusted mortality compared to patients not undergoing nephrectomy, which persisted with multivariate adjustment (HR 0.68, 95\% CI 0.63-0.74). The nephrectomy-treated patients also had a greater rate of retransplantation (incidence ratio 0.10 vs. $0.041, \mathrm{p}<$ 0.001 ). The average follow-up period was 2.93 years. Though this is an observational retrospective study, the authors remark in their discussion that there may be a beneficial effect of nephrectomy on mortality, likely related in part to the relatively low 30-day surgical mortality rate of $1.5 \%$ they observed. This point is very relevant in counseling patients, and it should be noted that this surgical data is more encouraging than previously published rates of mortality [36] and complications [38].

There is clinical data in support of the hypothesis that a graft remaining in situ can promote tolerance - as some authors describe it - or an immunosuppressive effect when stated more generally. In patients who had a PRA between 0 and $30 \%$ before their first transplant, graft nephrectomy is associated with a significantly less favorable PRA profile before a second transplantation (fig. 2) [36]. This counterintuitive association has led some to hypothesize that a failed graft is immunosuppressive. Interestingly, in patients with late graft failure, nephrectomy was associated with an increased risk of second graft loss (HR 1.20 , 95\% CI 1.02-1.41), while among those who had early graft failure, nephrectomy was associated with a decreased risk of second graft loss (HR 0.72, 95\% CI 0.56- 
Fig. 2. Observational data show that transplant nephrectomy is associated with a worsening PRA compared to no transplant nephrectomy. The paired columns along the horizontal axis show patients with $(\mathrm{N})$ and without $(\mathrm{NN})$ nephrectomy grouped by PRA 1-10, 11-30, 31-70, 71$100 \%$ prior to first transplant. Within each column, the distribution of patients according to PRA levels prior to repeat transplantation is shown. The $\mathrm{p}$ values shown compare first transplant with repeat transplantation [from 36, with permission].

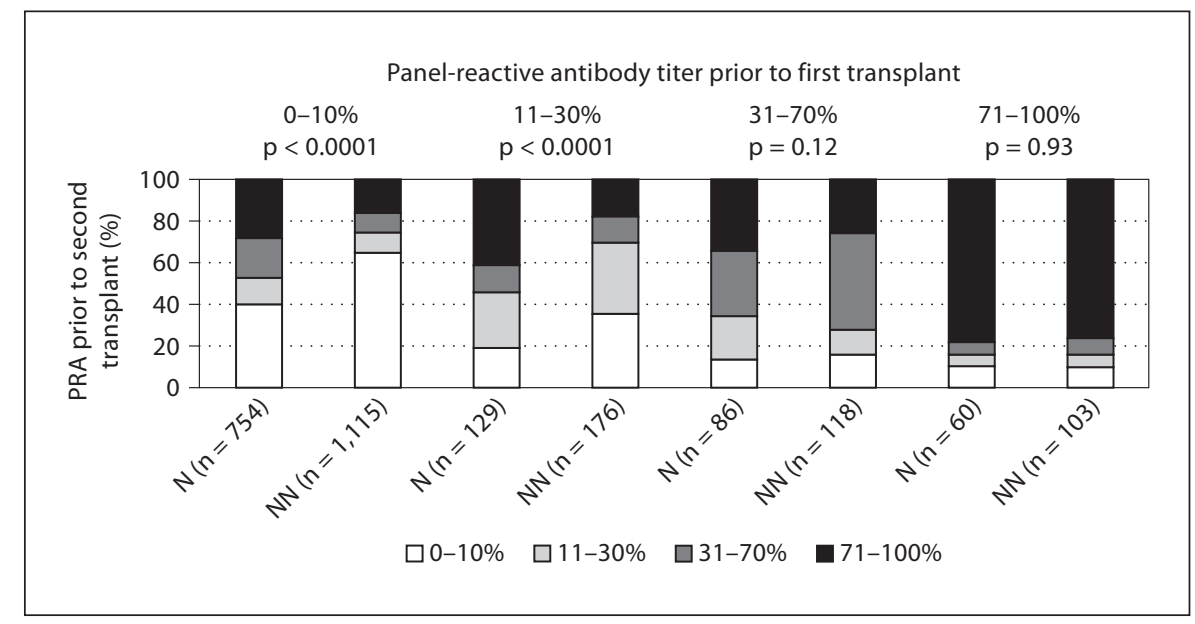

0.94) [36]. These figures are from uncontrolled observational data; one can only speculate on the clinical factors that may have led a patient to undergo graft nephrectomy.

In another observational study from Madrid, the authors compared 29 DAGL patients who underwent graft nephrectomy for traditional indications (fever, weight loss, anorexia, malaise) with 14 DAGL patients who lacked constitutional symptoms and did not undergo graft nephrectomy [39]. Hemoglobin, C-reactive protein, ferritin, and erythrocyte sedimentation rate were significantly worse in the first group of patients than in the nonnephrectomy patients. However, 6 months after nephrectomy, the former group of patients fared significantly better on a variety of laboratory parameters, including hemoglobin, albumin, erythropoietin resistance index, and C-reactive protein. These data suggest that there may be subclinical manifestations of an inflammation-malnutrition complex that accompanies a rejected organ, and that these abnormalities may be ameliorated by allograft nephrectomy.

In summary, the indications for allograft nephrectomy remain largely unclear, but the latest observational data suggest a salutary effect of removing a failed renal allograft. Data indicate that nephrectomy may be associated with either increased or decreased PRA indices, related to varying length of time between graft failure and graft removal. Nephrectomy is not a benign surgery based on reported complication rates. There may be a subclinical inflammatory syndrome associated with a non-functioning graft. The decision regarding allograft nephrectomy must be made on an individual basis for each patient, guided by the nuances of his or her clinical situation.

Care of the Patient after Renal Allograft Failure

\section{Conclusion}

Optimizing therapeutic options for the complex ESRD patient with a failed allograft is no easy task. This paper has attempted to synthesize data that will assist nephrologists in counseling their patients on the risks and optimal management of DAGL, the optimal approach to immunosuppression after graft loss, and the clinical risks and benefits of graft nephrectomy. A common thread running through these data is the survival advantage conferred by RRF [40], no matter which kidney - native or allograft - it is provided by. Complex clinical decisions such as these are unlikely to be addressed in randomized controlled trials, and this challenging dataset will improve only slowly and incrementally. In the meantime, nephrologists must continually study data in order to fully counsel their patients, and guide them through challenging decisions in an individualized manner.

\section{Disclosure Statement}

The authors have no conflicts of interest to disclose.

References

Am J Nephrol 2012;36:348-354 
-4 Ojo A, Wolfe RA, Agodoa LY, Held PJ, Port FK, Leavey SF, Callard SE, Dickinson DM, Schmouder RL, Leichtman AB: Prognosis after primary renal transplant failure and the beneficial effects of repeat transplantation: multivariate analyses from the united states renal data system. Transplantation 1998;66:1651-1659.

5 Meier-Kriesche HU, Kaplan B: Death after graft loss: a novel endpoint for renal transplantation. Transplant Proc 2001;33:34053406.

6 Gill JS, Rose C, Pereira BJ, Tonelli M: The importance of transitions between dialysis and transplantation in the care of end-stage renal disease patients. Kidney Int 2007;71: 442-447.

7 Gill JS, Abichandani R, Kausz AT, Pereira BJ: Mortality after kidney transplant failure: the impact of non-immunologic factors. Kidney Int 2002;62:1875-1883.

8 Meier-Kriesche HU, Baliga R, Kaplan B: Decreased renal function is a strong risk factor for cardiovascular death after renal transplantation. Transplantation 2003;75:12911295.

\9 Sasal J, Naimark D, Klassen J, Shea J, Bargman JM: Late renal transplant failure: an adverse prognostic factor at initiation of peritoneal dialysis. Perit Dial Int 2001;21:405410.

10 Davies SJ: Peritoneal dialysis in the patient with a failing renal allograft. Perit Dial Int 2001;21(suppl 3):S280-S284.

-11 Guo A, Mujais S: Patient and technique survival on peritoneal dialysis in the United States: evaluation in large incident cohorts. Kidney Int Suppl 2003,88:S3-S12.

$\checkmark 12$ Rao PS, Schaubel DE, Saran R: Impact of graft failure on patient survival on dialysis: a comparison of transplant-naive and postgraft failure mortality rates. Nephrol Dial Transplant 2005;20:387-391.

13 Rao PS, Schaubel DE, Jia X, Li S, Port FK, Saran R: Survival on dialysis post-kidney transplant failure: results from the scientific registry of transplant recipients. Am J Kidney Dis 2007;49:294-300.

-14 Perl J, Hasan O, Bargman JM, Jiang D, Na Y, Gill JS, Jassal SV: Impact of dialysis modality on survival after kidney transplant failure. Clin J Am Soc Nephrol 2011;6:582-590.

15 Gill JS: Managing patients with a failed kidney transplant: How can we do better? Curr Opin Nephrol Hypertens 2011;20:616-621.

$\checkmark 16$ Gill JS, Abichandani R, Khan S, Kausz AT, Pereira BJ: Opportunities to improve the care of patients with kidney transplant failure. Kidney Int 2002;61:2193-2200.

-17 Djamali A, Kendziorski C, Brazy PC, Becker $\mathrm{BN}$ : Disease progression and outcomes in chronic kidney disease and renal transplantation. Kidney Int 2003;64:1800-1807.
8 Baigent C, Landray MJ, Reith C, Collins R: The effects of lowering ldl cholesterol with simvastatin plus ezetimibe in patients with chronic kidney disease (study of heart and renal protection): a randomised placebocontrolled trial. Lancet 2011;377:2181-2192.

19 Opelz G, Wujciak T, Ritz E: Association of chronic kidney graft failure with recipient blood pressure. Collaborative Transplant Study. Kidney Int 1998;53:217-222.

20 Johnston O, Zalunardo N, Rose C, Gill JS: Prevention of sepsis during the transition to dialysis may improve the survival of transplant failure patients. J Am Soc Nephrol 2007;18:1331-1337.

21 Perl J, Wald R, McFarlane P, Bargman JM, Vonesh E, Na Y, Jassal SV, Moist L: Hemodialysis vascular access modifies the association between dialysis modality and survival. J Am Soc Nephrol 2011;22:1113-1121.

-22 Nassar GM, Ayus JC: Infectious complications of the hemodialysis access. Kidney Int 2001;60:1-13.

23 Akbari A, Hussain N, Karpinski J, Knoll GA: Chronic kidney disease management: comparison between renal transplant recipients and nontransplant patients with chronic kidney disease. Nephron Clin Pract 2007; 107:C7-C13.

24 Weyde W, Letachowicz W, Krajewska M, Golebiowski T, Letachowicz K, Kusztal M, Porazko T, Watorek E, Madziarska K, Klinger M: Arteriovenous fistula reconstruction in patients with kidney allograft failure. Clin Transplant 2008;22:185-190.

25 Wilmer WA, Pesavento TE, Bay WH, Middendorf DF, Donelan SE, Frabott SM, McElligott RF, Powell SL: Peritoneal dialysis following failed kidney transplantation is associated with high peritoneal transport rates. Perit Dial Int 2001;21:411-413.

26 Badve SV, Hawley CM, McDonald SP, Mudge DW, Rosman JB, Brown FG, Johnson DW: Effect of previously failed kidney transplantation on peritoneal dialysis outcomes in the Australian and New Zealand patient populations. Nephrol Dial Transplant 2006;21:776783.

27 Mujais S, Story K: Patient and technique survival on peritoneal dialysis in patients with failed renal allograft: a case-control study. Kidney Int Suppl 2006;103:S133-S137.

28 De Jonge $\mathrm{H}$, Bammens B, Lemahieu W, Maes BD, Vanrenterghem Y: Comparison of peritoneal dialysis and haemodialysis after renal transplant failure. Nephrol Dial Transplant 2006;21:1669-1674.
9 Schiffl H, Mucke C, Lang SM: Rapid decline of residual renal function in patients with late renal transplant failure who are re-treated with CAPD. Perit Dial Int 2003;23:398400.

30 Bernardo A, Fonseca I, Rodrigues A, Carvalho MJ, Cabrita A: Predictors of residual renal function loss in peritoneal dialysis: Is previous renal transplantation a risk factor? Adv Perit Dial 2009;25:110-114.

- 31 Jassal SV, Lok CE, Walele A, Bargman JM: Continued transplant immunosuppression may prolong survival after return to peritoneal dialysis: results of a decision analysis. Am J Kidney Dis 2002;40:178-183.

32 Scornik JC, Schold JD, Bucci M, Meier-Kriesche HU: Effects of blood transfusions given after renal transplantation. Transplantation 2009;87:1381-1386.

33 Lachmann N, Terasaki PI, Budde K, Liefeldt L, Kahl A, Reinke P, Pratschke J, Rudolph B, Schmidt D, Salama A, Schonemann C: Antihuman leukocyte antigen and donor-specific antibodies detected by luminex posttransplant serve as biomarkers for chronic rejection of renal allografts. Transplantation 2009;87:1505-1513.

34 Scornik JC, Meier-Kriesche HU: Blood transfusions in organ transplant patients: mechanisms of sensitization and implications for prevention. Am J Transplant 2011; 11:1785-1791.

- 35 Perl J, Bargman JM, Davies SJ, Jassal SV: Clinical outcomes after failed renal transplantation-does dialysis modality matter? Semin Dial 2008;21:239-244.

36 Johnston O, Rose C, Landsberg D, Gourlay WA, Gill JS: Nephrectomy after transplant failure: current practice and outcomes. Am J Transplant 2007;7:1961-1967.

- 37 Ayus JC, Achinger SG, Lee S, Sayegh MH, Go AS: Transplant nephrectomy improves survival following a failed renal allograft. J Am Soc Nephrol 2011;21:374-380.

38 Mazzucchi E, Nahas WC, Antonopoulos IM, Piovesan AC, Ianhez LE, Arap S: Surgical complications of graft nephrectomy in the modern transplant era. J Urol 2003;170:734737.

39 Lopez-Gomez JM, Perez-Flores I, Jofre R, Carretero D, Rodriguez-Benitez P, Villaverde M, Perez-Garcia R, Nassar GM, Niembro E, Ayus JC: Presence of a failed kidney transplant in patients who are on hemodialysis is associated with chronic inflammatory state and erythropoietin resistance. J Am Soc Nephrol 2004;15:2494-2501.

40 Bargman JM, Thorpe KE, Churchill DN: Relative contribution of residual renal function and peritoneal clearance to adequacy of dialysis: a reanalysis of the CANUSA study. J Am Soc Nephrol 2001;12:2158-2162. 